\title{
Emilia Romagna
}

Maria Caterina Cavallo ${ }^{(1)}$, Filippo Cipriani ${ }^{(2)}$, Simone Gerzeli ${ }^{(3)}$, Nadia Demarteau ${ }^{(4)}$, Alessia Marocco ${ }^{(2)}$, Francesco Bamfi ${ }^{(2)}$

\begin{abstract}
In Emilia Romagna region 77,6\% of women (aged 25-64) are screened regularly, meaning every 3 years. Considering regional tariffs and vaccine acquisition cost, the vaccination of 12 -year-old girls with a $90 \%$ coverage could prevent 51 cases of cervicocarcinoma and 22 related deaths and thus results to be cost-effective (29.903€/QALY). When the vaccination programme is extended to 16-year-old girls a further 47 cancer cases and 20 deaths could be prevented, with a very similar cost-effectiveness ratio. In Emilia Romagna region, the net cost for woman vaccinated is $177 €$ for the single cohort and $180 €$ for the multiple cohort.
\end{abstract}

Keywords: Emilia Romagna, HPV vaccination, economic \& clinical impact Farmeconomia e percorsi terapeutici 2008; 9 (Suppl. 1): 43-45

I dati specifici utilizzati nell'analisi per la regione Emilia Romagna sono rappresentati dalla demografia, dalla diffusione dello screening e dai costi degli interventi.

\section{RICORSO ALLO SCREENING}

La Tabella I riassume i dati più recenti per quanto riguarda il ricorso allo screening (programmi pubblici e screening privato) per la regione Emilia Romagna (tratti dall'Indagine Multiscopo ISTAT 2004-2005 [1]).

Lo screening è stato considerato "regolare" qualora la donna avesse dichiarato di essersi sottoposta a pap test almeno una volta negli ultimi tre anni; "irregolare" se l'ultimo pap test effettuato risultasse antecedente agli ultimi tre anni. A tale proposito nel modello si è assunto che il test venga effettuato solo 3 volte nell'arco della vita, con un intervallo di 10 anni e rispettivamente all'età di 30, 40 e 50 anni.

\section{COSTI}

In Tabella II si riassumono i dati di costo su base regionale dei vari interventi preventivi, di trattamento e follow-up.

Il costo dei trattamenti per le lesioni precancerose si basa sul costo medio pesato sulla base della frequenza dei trattamenti più frequenti. Il follow-up per gli anni successivi al trattamento è stato differenziato a seconda della severità della lesione: il follow-up per una CIN1 ha frequenza annuale e prevede il ricorso al pap test, visita ginecologica e colposcopia. Per le CIN23, il monitoraggio ha invece frequenza semestrale (in base a Linee guida nazionali [2] ed expert opinion). Per il costo del vaccino è stato utilizzato il prezzo ufficiale base di cessione al SS della regione Emilia Romagna.

\section{LA CAMPAGNA VACCINALE}

Tramite l'utilizzo del modello, si sono simulate due strategie di vaccinazione, in

\begin{tabular}{ll}
\hline \multicolumn{2}{c}{ Copertura screening } \\
\hline Regolare (ogni 3 anni) & $77,6 \%$ \\
Irregolare (> 3 anni) & $8,2 \%$ \\
Mai & $14,2 \%$ \\
\hline
\end{tabular}

\section{Tabella I}

Copertura dello screening (Dati ISTAT [1])

\begin{tabular}{|c|c|c|}
\hline Interventi & Oggetto & Costo \\
\hline \multirow{3}{*}{$\begin{array}{l}\text { Screening e trattamento CIN } \\
\text { (primo anno) }\end{array}$} & Screening negativo (pap test) & 14,25 \\
\hline & Trattamento CIN1 & 421,20 \\
\hline & Trattamento CIN23 & $1.866,38$ \\
\hline \multirow{2}{*}{$\begin{array}{l}\text { Follow-up delle CIN } \\
\text { (anni successivi) }\end{array}$} & Follow-up CIN1 & 43,60 \\
\hline & Follow-up CIN23 & 87,20 \\
\hline $\begin{array}{l}\text { Trattamento cancro } \\
\text { (complessivo) }\end{array}$ & $\begin{array}{l}\text { Cancro } \\
\text { (media pesata dei vari stadi) }\end{array}$ & 11.841 \\
\hline Vaccino & 1 ciclo completo di vaccinazione & $3 \times 83,90$ * \\
\hline
\end{tabular}

\section{Tabella II}

Costi regionali dei trattamenti (valori in euro)

* Prezzo d'aggiudicazione gara di Cervarix ${ }^{\top M}$ nella regione Emilia Romagna 


\begin{tabular}{lcc}
\hline \multicolumn{1}{c}{ Strategia vaccinale } & Coorte singola & Coorte multipla \\
\hline Coorte da vaccinare & $12 \mathrm{enni}$ & 12enni+16enni \\
Dimensione della coorte* & 16.328 & 32.603 \\
Copertura attesa & $90 \%$ & $90 \%$ \\
\hline
\end{tabular}

Tabella III

Caratteristiche della campagna vaccinale

* Valore approssimato delle ragazze emiliane nel 12esimo e 16esimo anno d'età (11enni e 15enni) al gennaio 2008 (dati ISTAT 2007) [3]

associazione allo screening, sul territorio regionale:

- una campagna mirata alla coorte singola delle donne di 12 anni, come da raccomandazione del SSN;

- una campagna multicoorte che comprende tanto le 12enni che le 16enni.

In entrambi i casi, si ipotizza un tasso di copertura atteso del $90 \%$. La dimensione com- plessiva delle coorti da vaccinare in Emilia Romagna, si basa sui dati ISTAT relativi alla popolazione residente al 1.1.2007 [3] (Tabella III).

\section{RISULTATI DELL'ANALISI FARMACOECONOMICA}

I benefici clinici e i risultati dell'analisi farmacoeconomica di Cervarix ${ }^{\mathrm{TM}}$ relativi alla vaccinazione delle 12enni associata allo screening, rispetto al solo screening, sono riportati in Tabella IV.

Nelle prime due colonne vengono riassunti i casi attesi in presenza del solo screening oppure in presenza di screening + vaccinazione; la differenza in termini assoluti rappresenta il beneficio clinico della vaccinazione della coorte delle 12enni, ovvero 51 casi di cervicocarcinoma evitati e 22 morti in meno. Nell'ultima colonna, è stato calcolato il Number Needed to Vaccinate (NNV) che rappresenta quanti soggetti occorre vaccinare per evitare un singolo

\begin{tabular}{lcccc}
\hline \multicolumn{1}{c}{ Benefici clinici } & Senza vaccinazione & Con vaccinazione & Differenza & NNV* \\
\hline Casi di cervicocarcinoma & 76 & 24 & 51 & 287 \\
Morti da cervicocarcinoma & 32 & 10 & 22 & 670 \\
Lesioni CIN23 & 754 & 334 & 420 & 35 \\
Lesioni CIN123 & 5.600 & 3.847 & 1.754 & 8 \\
\hline \multicolumn{1}{c}{ Risultati farmacoeconomici } & Senza vaccinazione & Con vaccinazione & Differenza & Rapporto incrementale \\
\hline Costi totali & 1.668 .563 & 4.867 .147 & 3.198 .585 & - \\
Anni di vita guadagnati & 490.227 & 490.307 & 80 & 40.017 (ICER) \\
QALY & 490.184 & 490.291 & 107 & 29.903 (ICUR) \\
\hline
\end{tabular}

\section{Tabella IV}

Coorte singola: benefici clinici e analisi economica (costi in euro)

* Numero di soggetti da vaccinare per evitare un singolo evento

- I risultati farmacoeconomici sono scontati annualmente del 3\%

Tutti i valori sono calcolati su cifre decimali e presentati come valori interi, salvo esplicite eccezioni

\begin{tabular}{lcccc}
\hline \multicolumn{1}{c}{ Benefici clinici } & Senza vaccinazione & Con vaccinazione & Differenza & NNV* \\
\hline Casi di cervicocarcinoma & 151 & 52 & 98 & 298 \\
Morti da cervicocarcinoma & 64 & 22 & 42 & 691 \\
\hline \multicolumn{1}{c}{ Risultati farmacoeconomici } & Senza vaccinazione & Con vaccinazione & Differenza & Rapporto incrementale \\
\hline Costi totali & 3.646 .657 & 10.218 .714 & 6.572 .057 & - \\
Anni di vita guadagnati & 965.578 & 965.739 & 161 & 40.930 (ICER) \\
QALY & 965.486 & 965.701 & 216 & 30.467 (ICUR) \\
\hline
\end{tabular}

\section{Tabella V}

Coorte multipla: benefici clinici e analisi economica (costi in euro)

* Numero di soggetti da vaccinare per evitare un singolo evento

- I risultati farmacoeconomici sono scontati annualmente del 3\%

Tutti i valori sono calcolati su cifre decimali e presentati come valori interi, salvo esplicite eccezioni 


\begin{tabular}{lcc}
\hline \multicolumn{1}{c}{ Strategia vaccinale } & Coorte singola & Coorte multipla \\
\hline Costo della vaccinazione (primo anno) & 3.698 .782 & 7.385 .558 \\
Costi diretti evitati dalla vaccinazione (lifetime) & 1.095 .797 & 2.110 .709 \\
Costo netto per donna vaccinata & $\mathbf{1 7 7}$ & $\mathbf{1 8 0}$ \\
\hline
\end{tabular}

evento: per il contesto della regione Emilia Romagna, 287 soggetti per evitare un caso di cervicocarcinoma. Considerando il rapporto incrementale tra costi e benefici (ICUR), l'adozione di Cervarix ${ }^{\mathrm{TM}}$ nella regione Emilia Romagna risulta costo-efficace.

La Tabella V, invece, riassume i risultati ottenibili dall'approccio a coorte multipla, che coinvolge sia le 12enni che le 16enni nella campagna vaccinale, confrontata con il solo screening.

Rispetto all'approccio a coorte singola, si riuscirebbero ad evitare ulteriori 47 casi di cancro e 20 decessi. Parte dell'efficienza della vaccinazione viene persa a causa di un numero maggiore di soggetti già infetti da HPV nelle 16 enni, come sottolineato dai valori crescenti degli NNV (298 vs 287 e 691 vs 670, rispettivamente). Tuttavia, ciò non va ad inficiare il rapporto di costo-efficacia che rimane favorevole.

L'impatto finanziario della vaccinazione sia in termini di spesa da sostenere per vaccinare una o due coorti che di ritorno in termini di costi medici diretti è mostrato in Tabella VI. La Tabella riporta, inoltre, il costo netto per donna vaccinata, ovvero quanto costa vaccinare una singola donna al netto dei costi diretti evitati con la vaccinazione stessa.

Occorre ricordare come il maggior impegno finanziario richiesto dalla strategia multicoorte sia richiesto solo per i primi 4 anni dall'inizio della vaccinazione, dal momento che al quinto anno di campagna le coorti si sono saldate e si continua a vaccinare solo le 12 enni.

\section{CONCLUSIONI}

In Emilia Romagna, l'introduzione della vaccinazione anti-HPV in aggiunta alla prassi corrente di screening, permetterebbe di evitare 51 casi di cervicocarcinoma e 22 morti correlate, per ogni coorte di 12enni vaccinate.

L'analisi farmacoeconomica di Cervarix ${ }^{\mathrm{TM}}$ ha evidenziato un buon profilo di costo-efficacia, per la coorte delle 12enni. L'esempio dell'offerta di vaccinazione allargata alle 16enni rileva l'impatto clinico e la convenienza economica anche per coorti multiple.

In Emilia Romagna, il costo netto per donna vaccinata è di $€ 177$ per la coorte singola e $€ 180$ per la coorte multipla.
Tabella VI Impatto sul budget (costi in euro)

\section{BIBLIOGRAFIA}

1. Indagine Multiscopo ISTAT 2004-2005. Disponibile online http://www.istat.it/dati/dataset/20080131_00/

2. Gestione della paziente con pap test anormale. Linee Guida Edizione 2006 a cura della Società italiana di Colposcopia e Patologia Cervico Vaginale. La Colposcopia in Italia. Anno XXI, N.1, Dicembre 2006

3. Demografia ISTAT. Disponibile online www.demo.istat.it 